\title{
Sore throat as a side effect of abobotulinum toxin A injection for upper limb spasticity after stroke: A case report
}

\author{
Esra Giray (D), Evrim Karadag-Sayg1 (D) \\ Department of Physical Medicine and Rehabilitation, Marmara University School of Medicine, Istanbul, Turkey \\ Received: April 05, 2018 Accepted: July 12, 2018 Published online: March 03, 2020
}

\begin{abstract}
Despite sore throat exists in the product characteristics of abobotulinum toxin A as one of the side effects, no available reports of sore throat after abobotulinum toxin A injection for adult upper limb spasticity could be found in the literature to guide physicians for the management. Herein, we report a case of poststroke spasticity who develop sore throat immediately after abobotulinum toxin A (Dysport ${ }^{\circ}$ ) injection of forearm muscles which completely resolved after observation for eight hours. This case report highlights a rare side effect of abobotulinum toxin A and provides information regarding the follow-up process of side effects which would guide physicians for the management.
\end{abstract}

Keywords: Adverse drug event, botulinum toxins type A, sore throat, spasticity.

Botulinum toxin injections are usually applied as focal antispastic agents as the part of rehabilitation programs to improve active and passive function, to decrease pain related to passive mobilization and painful spasms, to improve hygiene, and to avoid contractures. Botulinum toxin inhibits the release of acetylcholine from nerve terminals at the neuromuscular junction and weakens the muscle and, thus, decreases spasticity in this way. Botulinum toxin A has been shown to be a safe and effective therapy for the treatment of upper limb spasticity after stroke. ${ }^{[1]}$ However, several side effects related to spread of abobotulinum toxin A distant from the site of administration have been mentioned in the summary of product characteristics. ${ }^{[2]}$ These side effects are muscle weakness, dysphagia, skin rashes, flu-like symptoms, local injection site pain, asthenia, nasopharyngitis, urinary tract infections, dizziness, and epileptic seizures. ${ }^{[2]}$ Although sore throat and throat irritation exist as a side effect in the summary of product characteristics, these side effects are reported in neither safety trials for adult upper limb spasticity nor case reports. No information about the follow-up process of these side effects can be found in the literature which may help to aid clinicians to manage the side effects and provide patient information.

Herein, we report a case of sore throat or throat irritation as a side effect of abobotulinum toxin A for post-stroke spasticity and completely resolved after observation for eight-h in the emergency department.

\section{CASE REPORT}

A 55-year-old female patient with a history of ischemic stroke eight years ago was admitted to the outpatient clinic of neurological rehabilitation with a complaint of clenched fist with difficulty in opening the fingers and the thumb and foul smell of palm. She had a history of early menopause (at the age of 42 years), diabetes mellitus, essential hypertension, hypercholesterolemia, and iatrogenic hypothyroidism (total thyroidectomy). She was already receiving $2.5 \mathrm{mg}$ of ramipril and $12.5 \mathrm{mg}$ of hydrochlorothiazide, $20 \mathrm{mg}$ of atorvastatin, $2 \mathrm{mg}$ of glimepiride, and $100 \mu \mathrm{g}$ of levothyroxine sodium. She received repeated abobotulinum toxin $\mathrm{A}$ injection for two times previously as clinically indicated. On physical examination, she was cognitively intact and 
presented with severe spasticity in the upper extremity. The degree of spasticity of her left elbow flexors, forearm pronators, wrist flexors, finger flexors, and thumb flexor was 3/4 using the Modified Ashworth Scale (MAS). Botulinum toxin injections in the upper extremity was intended to decrease pain related to hypertonicity, to improve hand hygiene, to ease neurorehabilitative interventions, to ease wearing splints, and to prevent from contractures. After she received a total dose of $500 \mathrm{IU}$ of abobotulinum toxin A (200 IU for biceps and $100 \mathrm{IU}$ per flexor digitorum superficialis, flexor digitorum profundus and pronator muscles), she complained about severe pain and discomfort at the region corresponding to the middle of the neck right above the sternum. Botulinum toxin injection was discontinued immediately, and the patient was monitored. Physical examination revealed a body temperature of $36.6^{\circ} \mathrm{C}$, oxygen saturation of $90 \%$ on room air, blood pressure of $150 / 90 \mathrm{mmHg}$, pulse rate of $80 \mathrm{bpm}$, blood glucose level of $200 \mathrm{mg} / \mathrm{dL}$, and respiratory rate of 20 breaths/min. Her pupils were equal and reactive. Motor and sensory examination and deep tendon reflexes were normal. She was able to swallow her saliva. Due to a suspected myocardial infarction, iatrogenic botulism and the possibility of respiratory muscle involvement over time, she was transferred to the emergency department and observed for eight $h$. The electrocardiogram (ECG) did not show ischemic changes. The cardiac enzymes were within normal limits. All routine laboratory test results were in the normal range. During close observation in emergency department, ECG and cardiac enzymes were repeated and revealed normal results. Finally, her symptoms gradually diminished and disappeared, and eight hour after the injection, she had no residual symptoms and was in a good health state. She was discharged from the emergency department after eight hour follow-up. She was instructed to revisit the emergency department immediately, if she had difficulty in breathing or swallowing. No additional symptoms occurred within the following three weeks. A written informed consent was obtained from the patient.

\section{DISCUSSION}

In the presented case, we described a patient who developed sore throat immediately after abobotulinum toxin A injection for upper limb spasticity following stroke in the setting of injections at therapeutic dosage. The risk for development distant spread of toxin effects following therapeutic botulinum neurotoxin A injections could be related to several factors: age, sex, total injected dose, injected dose based on body weight $(\mathrm{U} / \mathrm{kg})$, injection frequency, botulinum toxin serotype, or underlying comorbidities. A review of the literature and reported cases revealed that risk of developing systemic effects did not appear to be associated with dose based on the body weight. The risk of occurrence of systemic effect may be related to the total injection dose and the frequency of injections. ${ }^{[3]}$ The risk is probably the highest in children who were treated, but also they can happen in adults treated for spasticity, particularly in the presence of other underlying predisposing conditions. Side effects which are associated with the spread of effect have been reported at doses lower than the maximum recommended total dose. The present case had the third injection with a dose of therapeutic range. She tolerated previous abobotulinum toxin A injections, making this side effect nearly impossible to predict.

Reported throat problems after abobotulinum toxin A injection are dry throat, sore throat, and throat irritation. Throat problems as a side effect after abobotulinum toxin A for upper limb spasticity after stroke have not been reported previously, while there have been several reports for indications other than upper limb spasticity and with other serotypes of botulinum toxin. Sore throat after receiving abobotulinum toxin $\mathrm{A}$ for cervical dystonia and dysphonia due to adductor spasm was defined previously. ${ }^{[4,5]}$

The mechanism of sore throat or throat irritation side effect of botulinum neurotoxin $\mathrm{A}$ has yet to be defined. Botulinum neurotoxin $\mathrm{A}$ is a powerful and a neurotoxin produced by the bacterium Clostridium botulinum. The active form of botulinum toxin is a polypeptide consisting of a heavy chain $(100 \mathrm{kDa})$ and light chain $(50 \mathrm{kDa})$. Botulinum neurotoxin $\mathrm{A}$ acts by binding presynaptically to high-affinity recognition sites on the cholinergic nerve terminals and reducing by permitting acetylcholine release from nerve terminals at the neuromuscular junction resulting in a neuromuscular blocking effect. Botulinum neurotoxin A binds to an acceptor protein and is endocytosed at the presynaptic membrane of acetylcholine nerve terminals. Through the action of the $\mathrm{N}$-terminal on the heavy-chain, a pore is formed on the endocytic membrane, which inhibits the release of the light chain into the cytosol. This light chain, which is a zinc protease, performs the key action of botulinum neurotoxin $\mathrm{A}$, by cleaving soluble $\mathrm{N}$-ethylmaleimidesensitive factor attachment receptor proteins (SNARE proteins). The SNAREs are docking proteins for acetylcholine vesicles which allow for the release of 
acetylcholine into the synaptic cleft. As the fusion of the vesicle membranes becomes inhibited, a temporary blockade of acetylcholine release occurs at cholinergic synapses, leading to a local chemodenervation. Transient synapses are consequently formed by axonal sprouting. Botulinum neurotoxin $\mathrm{A}$ act at four different sites in the body: the neuromuscular junction, autonomic ganglia, postganglionic parasympathetic nerve endings and postganglionic sympathetic nerve endings which release acetylcholine. Botulinum neurotoxin A shows its classical efficacy of decreasing muscle tone at neuromuscular junction. Botulinum neurotoxin A also exhibits blocking effects on autonomic cholinergic transmission. Therefore, the patients with botulism frequently present autonomic symptoms such as reduced saliva secretion and lacrimation, as well as constipation, altered micturition and cardiovascular regulation, delayed pupillary reaction to light, mydriasis, and accommodation paresis. ${ }^{[4]}$ Botulinum neurotoxin A binds to peripheral cholinergic nerve terminals of the neuromuscular junction as well as sympathetic, parasympathetic, and postganglionic terminals. ${ }^{[4]}$ Thus, it is not specific to the neuromuscular junction and has the effect to prevent cholinergically transmission of autonomic fibers. Localized botulinum neurotoxin A injection disrupts cholinergic transmission and has potential to cause focal dysautonomia. Mucociliary transport and laryngeal secretions are thought to be mediated in part by autonomic, cholinergic transmission. Throat irritation/sore throat may be due to disrupted autonomic cholinergic transmission to submucosal glands of the airway or mucociliary clearance leading to burning, tickling, or irritation of the larynx/throat or dry throat/mouth. ${ }^{[4]}$

Iatrogenic botulism and acute coronary syndrome are differential diagnoses of throat irritation or sore throat after botulinum neurotoxin A. These two lifethreatening conditions should be considered in the differential diagnosis. It is important to recognize and differentiate these side effects from iatrogenic botulism. Incubation period of botulism is between six $\mathrm{h}$ to eight days. Blurred vision, dysphagia, and dysarthria are common initial complaints of botulism. Throat irritation/sore throat are similar symptoms with dysphagia. Sore throat can be differentiated from botulism with the absence of muscle weakness and cranial nerve palsy. Also, experienced pain in the chest or throat can be so intense that clinicians may confuse it with a heart attack. However, it is not possible to differentiate the two life-threatening diagnosis from sore throat without follow-up.
To find out the association between sore throat and abobotulinum toxin A treatment we used Naranjo Scale. The Naranjo Scale is a questionnaire designed by Naranjo et al. ${ }^{[6]}$ to identify whether an adverse drug reaction is actually associated with the drug use rather than other factors. The temporal association of drug use and adverse event development, alternative causes for the event, drug levels, dose-response association and previous patient experience with the medication are questioned and probability score is calculated. ${ }^{[6]}$ This case scored 5 on the Naranjo Scale, showing a possible causality.

Sore throat or throat irritation related to abobotulinum toxin A injection for spasticity after stroke has not been reported previously. To the best of our knowledge, this is the first case report of sore throat occurring immediately after abobotulinum toxin A injection for upper arm spasticity following stroke.

In conclusion, despite use of therapeutic and low doses, local botulinum neurotoxin A injection may lead to sore throat. Sore throat/throat irritation may cause anxiety in both patients and clinicians. Clinicians should be aware of this side effect and its differential diagnosis to manage the side effect and inform the patient about the temporary and benign nature of the side effect. The mechanism of sore throat should be highlighted to find a symptomatic cure and whether adverse event reappears, when the drug is readministered.

\section{Declaration of conflicting interests}

The authors declared no conflicts of interest with respect to the authorship and/or publication of this article.

\section{Funding}

The authors received no financial support for the research and/or authorship of this article.

\section{REFERENCES}

1. Gracies JM, Brashear A, Jech R, McAllister P, Banach M, Valkovic P, et al. Safety and efficacy of abobotulinumtoxin A for hemiparesis in adults with upper limb spasticity after stroke or traumatic brain injury: a double-blind randomised controlled trial. Lancet Neurol 2015;14:992-1001.

2. Available at: https://dysporthcp.com/docs/pdfs/Dosing_ and_Dilution_Brochure.pdf.

3. Crowner BE, Torres-Russotto D, Carter AR, Racette BA. Systemic weakness after therapeutic injections of botulinum toxin a: a case series and review of the literature. Clin Neuropharmacol 2010;33:243-7.

4. Fisher KV, Giddens CL, Gray SD. Does botulinum toxin alter laryngeal secretions and mucociliary transport? J Voice 1998;12:389-98. 
5. Comella CL, Jankovic J, Shannon KM, Tsui J, Swenson M, Leurgans S, et al. Comparison of botulinum toxin serotypes $A$ and $B$ for the treatment of cervical dystonia. Neurology 2005;65:1423-9.
6. Naranjo CA, Busto U, Sellers EM, Sandor P, Ruiz I, Roberts EA, et al. A method for estimating the probability of adverse drug reactions. Clin Pharmacol Ther 1981;30:239-45. 